\title{
Electric-Field-Driven Direct Desulfurization
}

Bogdana Borca, ${ }^{1,2{ }^{*}}$ Tomasz Michnowicz, ${ }^{1 \neq}$ Rémi Pétuya, ${ }^{3: \#}$ Marcel Pristl, ${ }^{1}$ Verena

Schendel, ${ }^{1 \dagger}$ Ivan Pentegov, ${ }^{1}$ Ulrike Kraft, ${ }^{1 \perp}$ Hagen Klauk, ${ }^{1}$ Peter Wahl, ${ }^{1,4}$ Rico Gutzler, ${ }^{1}$

Andrés Arnau, ${ }^{3,5}$ Uta Schlickum ${ }^{1 *}$ and Klaus Kern ${ }^{1,6}$

${ }^{1}$ Max Planck Institute for Solid State Research, 70569 Stuttgart, Germany,

${ }^{2}$ National Institute of Materials Physics, 077125 Măgurele-Ilfov, Romania.

${ }^{3}$ Donostia International Physics Centre, E-20018 Donostia - San Sebastián, Spain,

${ }^{4}$ SUPA, School of Physics and Astronomy, University of St Andrews, North Haugh, St

Andrews, KY16 9SS, United Kingdom,

${ }^{5}$ Departamento de Física de Materiales UPV/EHU and Material Physics Center (MPC),

Centro Mixto CSIC-UPV/EHU, E-20018 Donostia - San Sebastián, Spain,

${ }^{6}$ Institut de Physique, École Polytechnique Fédérale de Lausanne (EPFL), CH-1015

Lausanne, Switzerland.

ABSTRACT. The ability to elucidate the elementary steps of a chemical reaction at the atomic scale is important for the detailed understanding of the processes involved which is key to uncover avenues for improved reaction paths. Here, we track the chemical pathway of an irreversible direct desulfurization reaction of tetracenothiophene adsorbed on the $\mathrm{Cu}(111)$ closed-packed surface at the submolecular level. Using the precise control of the tip position 
in a Scanning Tunneling Microscope and the electric field applied across the tunnel junction, the two carbon-sulfur bonds of a thiophene unit are successively cleaved. Comparison of spatially mapped molecular states close to the Fermi level of the metallic substrate acquired at each reaction step with density functional theory calculations reveals the two elementary steps of this reaction mechanism. The first reaction step is activated by an electric field larger than $2 \mathrm{~V} \mathrm{~nm}^{-1}$, practically in absence of tunneling electrons, opening the thiophene ring and leading to a transient intermediate. Subsequently, at the same threshold electric field and with simultaneous injection of electrons into the molecule the exergonic detachment of the sulfur atom is triggered. Thus, a stable molecule with a bifurcated end is obtained, which is covalently bound to the metallic surface. The sulfur atom is expelled from the vicinity of the molecule.

KEYWORDS: tetracenothiophene, desulfurization, electric field, single molecules, STM, DFT.

Chemical reactions implying the dissociation of carbon-sulfur bonds are fundamental for understanding the carbon and sulfur cycles in nature. ${ }^{1-3}$ Moreover, methods for removing the sulfur atoms from organic molecules, the desulfurization reactions, are of industrial significance in processes aimed at cleaning natural fuels. ${ }^{4}$ A popular method is based on the use of catalysts to diminish the activation energy of the reaction. ${ }^{5}$ Indeed, when released, sulfur compounds may have a detrimental effect on the natural environment. ${ }^{6}$ A clear understanding of the mechanism of the reaction by local control down to the submolecular level may help to identify more efficient reaction paths. 
Scanning Tunneling Microscopy (STM) constitutes an adequate tool to trigger and analyze chemical reactions with atomic precision. The formation, ${ }^{7-10}$ scission, ${ }^{11-17}$ or deformation of chemical bonds ${ }^{18-23}$ and intramolecular atomic rearrangements ${ }^{24-27}$ have been activated on individual molecules using the STM. Working at low temperatures transient intermediate states have also been resolved. ${ }^{28}$ Chemical reactions can be stimulated by the STM electronically with the tunneling current, through the applied electric field between tip and sample, or by a combination of both mechanisms. The tunneling electrons excite molecular states that participate in the formation of chemical bonds or activate vibrational modes and, thus, produce molecular changes associated with chemical reactions. ${ }^{7-11,15,20,24}$ The electric field confined between the tip apex and the substrate has been shown to stimulate conformational transitions, ${ }^{18,19}$ complex movements of molecules ${ }^{25-27}$ or to trigger dissociation reactions. ${ }^{16,17}$ Additionally, it was shown recently in a STM-break junction setup that the electric field can be used to obtain a catalytic-like effect for the acceleration of a Diels-Alder reaction, ${ }^{29}$ which highlights the potential of oriented electric fields to control chemical processes. ${ }^{30}$

In our study, the electric field is employed to trigger locally a desulfurization process on a single molecular subunit, a thiophene ring, part of a tetracenothiophene (TCT) molecule. The spatial mapping of the molecular states close to the Fermi energy of the substrate, complemented with density functional theory (DFT) calculations, enables us to identify the two elementary steps of the direct desulfurization reaction, i.e., the successive cleavage of the two $\mathrm{S}-\mathrm{C}$ bonds. 


\section{RESULTS AND DISCUSSION}

To address locally the thiophene moiety of the TCT molecules (chemical structure in Figure 1b), a sub-monolayer amount of TCT was deposited on the closed-packed copper $\mathrm{Cu}(111)$ surface held at $200 \mathrm{~K}$. At lower temperatures (6 K), individual molecules are imaged by the STM as asymmetric "dumbbell"-shaped protrusions (Figure 1a). The thiophene unit appears brighter, showing a higher density of states compared to the tetracene part (for a detailed structural analysis see SI - adsorption conformation). The desulfurization reaction is activated by precisely positioning the STM tip apex on top of the thiophene ring and applying as stimulus a short voltage pulse that creates a strong electric field in the junction and injects electrons into the system (Figure 1b). Each step of the chemical reaction sequence is imaged by STM with noninvasive tunneling parameters that do not affect the molecular configuration. Two irreversible reaction pathways, differing by the action of the applied stimulus, are successfully activated locally converting single molecules from the initial state 1 to the final state 2 (Figure 1a,c).

a)

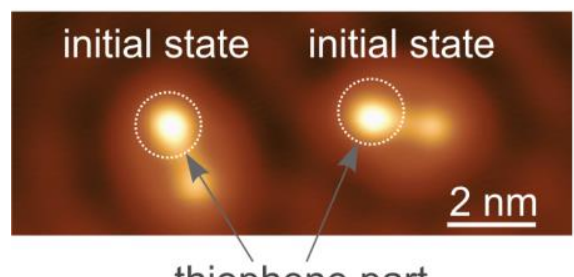

thiophene part b)
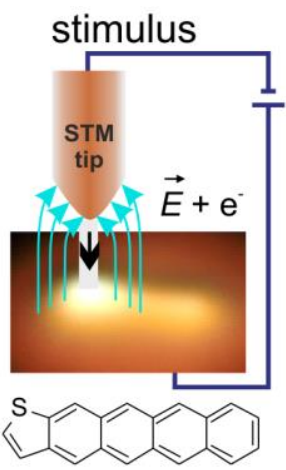

c)

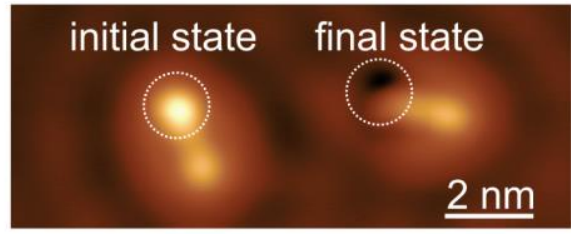

Figure 1. STM investigation of the desulfurization reaction on single TCT molecules adsorbed on $\mathrm{Cu}(111)$. a) $\mathrm{STM}$ topographic image $(I=100 \mathrm{pA}, V=100 \mathrm{mV})$ before the reaction showing two TCT molecules in initial conformation 1. b) Schematic representation of the STM-induced desulfurization reaction on a single molecule initiated by the electric field $\vec{E}$ (the field lines are symbolized by the cyan arrows) and the tunneling electrons $\mathrm{e}^{-}$(the 
current flow is symbolized by the black arrow). Bottom panel: chemical structure of the TCT molecule. c) STM image after the reaction was induced in the molecule at the right part of panel a). This molecule is now in the final conformation 2. The white circles indicate the thiophene group of the molecule before and after the reaction.

One pathway, which we call $\mathbf{R}$, leads to a direct transition from the initial state $\mathbf{1}$ to the final state 2. For the second pathway we can follow a two-step reaction, where we call the individual steps $\mathbf{R I}$ and $\mathbf{R I I}$. The end-products of $\mathbf{R I}+\mathbf{R I I}$ and of $\mathbf{R}$ are equivalent as demonstrated in Figure 2, and thus $\mathbf{R}=\mathbf{R} \mathbf{I}+\mathbf{R} I \mathbf{I}$, as we will show in detail later. Placing the tip above the thiophene ring of $\mathbf{1}$ (Figure 2a) at a large relative tip-molecule distance of 2-3 $\mathrm{nm}$ and applying a voltage above $5.5 \mathrm{~V}$ to the sample an intermediate state $1 \mathrm{i}$ is reached. The definition of the relative tip-molecule distance is described in the methods section. The induced reaction results in changes of the molecular structure corresponding to a reduced density of states at the thiophene site and, in addition, to the appearance of a dark spot at the initial position of the sulfur atom (Figure 2a, central panel). For the second step RII the relative distance between the tip and the thiophene ring was reduced to values smaller than $1.25 \mathrm{~nm}$ while applying a voltage pulse between 1.5 and $4.5 \mathrm{~V}$, depending on the distance. Again, a drastic change is observed in the topographic appearance at the reacted side of the molecules, where the density of states is further reduced. The images in Figure 2a are acquired with a metal tip that does not allow resolving the molecular orbitals.

A comparison between STM images taken with a functionalized tip showing the orbital structure of the molecules and DFT calculations using van-der-Waals density functional in which exchange and correlations are described consistently (vdW-DF-cx) $)^{31,32}$ reveals the detailed reaction pathway of the desulfurization reaction mechanism. Figure $2 \mathrm{~b}$ shows the spatial mapping of the molecular state located at the Fermi energy of the reactant, 
intermediate and product of this two-step reaction. These images are acquired in constant height mode using a functionalized molecular tip. The functionalization is performed by standard vertical manipulation to place a TCT molecule at the tip apex. The initial configuration 1 (Figure $2 \mathrm{~b}$ - left panel) resembles the lowest unoccupied molecular orbital in the gas phase, gas-phase-LUMO, of TCT (see SI - electronic structure) and is comparable to the calculated STM appearance obtained within the Tersoff-Hamann approximation (Figure $2 \mathrm{c}-$ left panel). ${ }^{33}$ This observation is traced back to a strong hybridization at the interface and to a charge transfer from the substrate to the molecule, a process which shifts the energy of this orbital, named here surface-modified-LUMO towards the Fermi level, similar to the behavior of the chemical analogue anthradithiophene when adsorbed on $\mathrm{Cu}(111) .{ }^{34} \mathrm{The}$ simulated STM images are in qualitative agreement with the experimental topographies (Figure $2 \mathrm{~b}$ and $\mathrm{c}$, center and right panels). In the STM images, the intermediate, 1i, and final, 2, conformations appear with a depression in close vicinity of the molecule (Figure $2 \mathrm{a}$ and $\mathrm{b}$, center and right panels). This feature is reproduced by the calculations and is in the location of the former $\mathrm{S}$ position in the intermediate state $\mathbf{1 i}$ and the final state $\mathbf{2}$. The calculated molecular structures, plotted in a ball-and-stick representation in Figure 2d, provide snapshots of the atomic structure of the molecules-substrate system along the reaction pathway. Based on these experimental and theoretical evidences we exclude conformational changes related to lateral translation or the scission of other chemical bonds, as described in detail in the Supporting Information. 


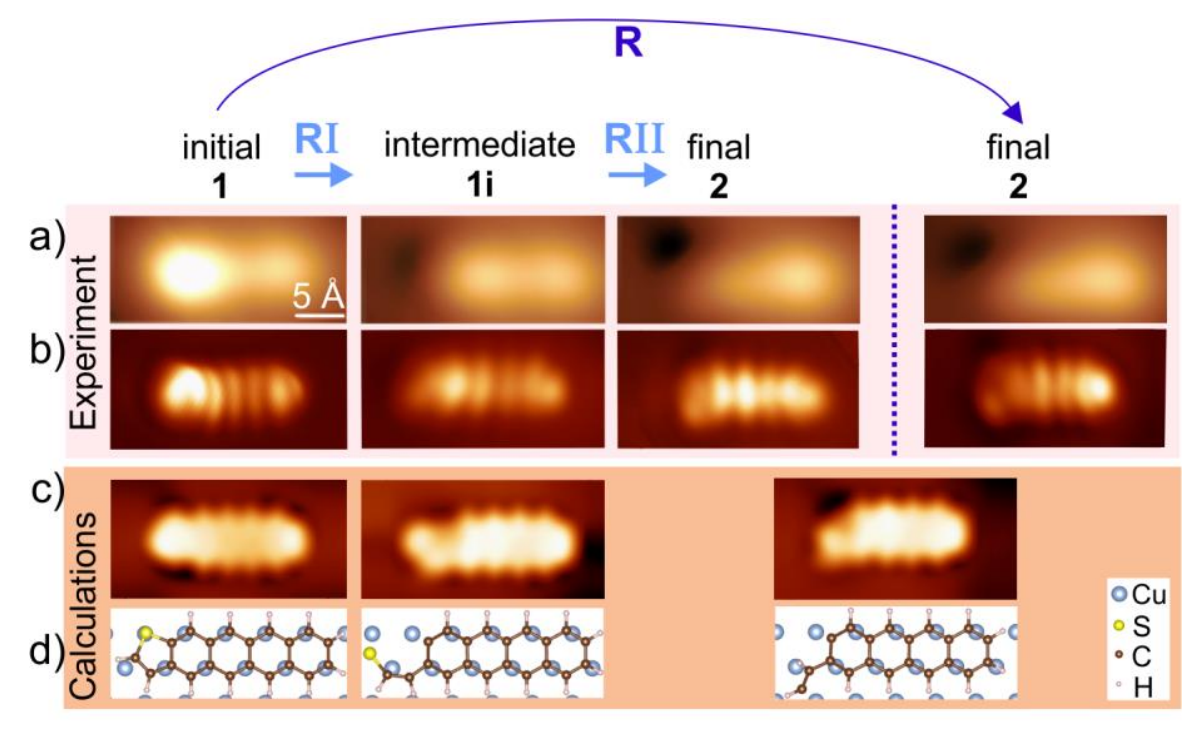

Figure 2. Molecular conformations at each stage of the direct desulfurization reaction obtained by STM investigations and DFT calculations. a) Topographic images acquired in constant current mode. The reaction $\mathbf{R}$ represents the reaction that directly transforms the system from state $\mathbf{1}$ to the final state $\mathbf{2}$. The reactions RI and RII represent the two steps of the desulfurization process, from the initial state $\mathbf{1}$ to the intermediate state $\mathbf{1 i}$ and from $\mathbf{1 i}$ to the final state 2. b) Spatial maps of the molecular states obtained with a functionalized tip, acquired in constant height mode $(I=100 \mathrm{pA}, V=-1 \mathrm{~V}$ left image, $I=100 \mathrm{pA}, V=100 \mathrm{mV}$ all other images). c) Molecular appearances in the Tersoff-Hamann approximation obtained from DFT-vdW simulations for each molecular state. d) Ball-and-stick representation of the calculated molecular sequences on the $\mathrm{Cu}(111)$ surface (top views).

The analysis reveals that the RI reaction consists of the scission of one of the two carbonsulfur bonds, opening the thiophene ring (Figure $2 \mathrm{~d}$ ). The cleaved bond results in a geometric reorganization of the two carbon atoms of the thiophene ring, increasing angles between $\mathrm{C}-\mathrm{C}$ bonds, which leads to the accommodation of the sulfur atom at a new position. When the second carbon-sulfur bond is dissociated in RII, the system releases energy and, thus, the sulfur is repelled from the surrounding of the molecule. This step is accompanied by further 
repositioning of the two carbon atoms. Therefore, the entire process encompasses cleavage of the $\mathrm{C}-\mathrm{S}$ bonds, new bond formations between the opened thiophene ring and the surface, distortions and translation of the S-atom.

Next, we discuss the experimental procedure used to deduce the nature of the stimuli which trigger the desulfurization reaction. At large tip-sample distances the dominating stimulus is the electric field present in the tunneling junction when a bias voltage is applied. By decreasing the tip-sample distance, the tunneling current increases and, thus, more electrons are injected into the system. In our analysis we have been able to correlate the two reaction steps RI and RII with two different regimes. To trigger and to track the reaction RI we performed detailed studies of the experimental parameters required to initiate the reaction. We first acquired a topographic image of the molecule in state $\mathbf{1}$ and then stabilized the tip above the thiophene moiety at the tunneling parameters $I=100 \mathrm{pA}$ and $U=100 \mathrm{mV}$. After disabling the feedback loop that controls the tip height, we increased the tip molecule distance $z$ to about $2.2 \mathrm{~nm}(z=0$ corresponds to a tunnel resistance of $16.5 \pm 1.2 \mathrm{M} \Omega)$ and applied a short voltage pulse of $5 \mathrm{~V}$ (minimum duration $100 \mu \mathrm{s}$ ). At these large tip-sample distances the current was in the noise limit of our current amplifier. This observation leads us to conclude that the dominant stimulus of RI is the electric field. It transforms the system from the initial to the intermediate state. The successful transition to the intermediate state 1i is confirmed afterwards by topographic imaging.

The second step RII can only be activated at much shorter distances between tip and thiophene ring. To trigger RII, we placed the STM tip on top of the thiophene unit at a distance stabilized by the tunneling parameters $(I=100 \mathrm{pA}, U=100 \mathrm{mV})$ and we switched the feedback loop off. Subsequently, we measured at various tip-sample distances $z$ (between 0 and $1.25 \mathrm{~nm}$ relative distances) the transitions $\mathbf{R I I}$ (1i-2 path) that we could detect in the 
voltage-dependent current traces by recording $I(V)$ curves (Figure 3a). At these distances we have a measurable current and we can detect a sudden decrease of the current as a step-like feature in the $I(V)$ traces. This step-like feature coincides with a change in the local density of states at the submolecular level. This abrupt change indicates the transition from state 1i to the finale state $\mathbf{2}$, as identified subsequently in the STM images. The current and the voltage values for which the reaction is stimulated are marked with $\left(I_{R}, V_{R}\right)$. The voltages $V_{R}$ which trigger the pathway RII follow a linear dependence with $z$ (Figure $3 \mathrm{~b}$ solid circles). This is a characteristic behavior of an electric-field-driven reaction. ${ }^{18,19}$ The slope of this linear dependence is about $2 \mathrm{Vnm}^{-1}$ and represents the critical electric field necessary to trigger the reaction. Hence, the electric field is the stimulus that activates the pathway RII but the necessity to work at short tip-thiophene distances to run this reaction step ensures the injection of electrons into the molecular system (see Figure 3b). In Figure 3c the $\left(I_{R}, V_{R}\right)$ values at which reaction $\mathbf{R I}$ is activated are plotted. It is observed that these values follow exactly the linear dependence extracted in Figure 3b. If we work in the regime of short tipsample distances, we can directly transform the system from the initial state $\mathbf{1}$ to the final state $\mathbf{2}$ (pathway $\mathbf{R}$ ), since we apply at the same time a sufficiently high electric field and inject a sufficiently high amount of electrons into the system to break both $\mathrm{C}-\mathrm{S}$ bonds. On the time scale of the measurement we cannot observe the intermediate product. We observe exactly the same end-product for RI+ RII that we observe for $\mathbf{R}$ (see Figure 2). When a functionalized tip was used (Figure 2b), the molecules in the final state have the same molecular orbital shape, especially at the reacted side of the molecule, although the relative intensities of different images vary slightly due to different conformations of the molecule attached to the tip apex. In addition, the corresponding $\left(I_{R}, V_{R}\right)$ values are very similar to the values obtained for RII (open squares in Figure $3 b$ ). On the basis of these arguments we conclude that $\mathbf{R}=\mathbf{R} \mathbf{I}+\mathbf{R I I}$. 
Therefore, a critical field of about $2 \mathrm{~V} \mathrm{~nm}^{-1}$ has to be overcome to activate the reaction pathways, RI and RII. A key difference between the reaction paths lies in the activation mechanism. From the analysis, we conclude that RI is triggered only by the electric field, whereas RII is driven by a combination of the electric field and the injection of electrons. The direct desulfurization reaction, including all pathways, can be activated only with a positive voltage polarity highlighting the importance of the electric field.

a)

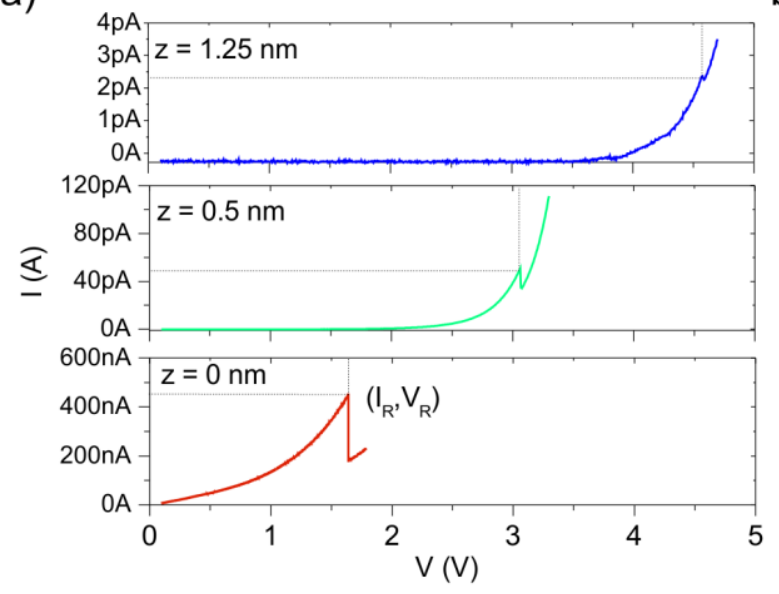

b)

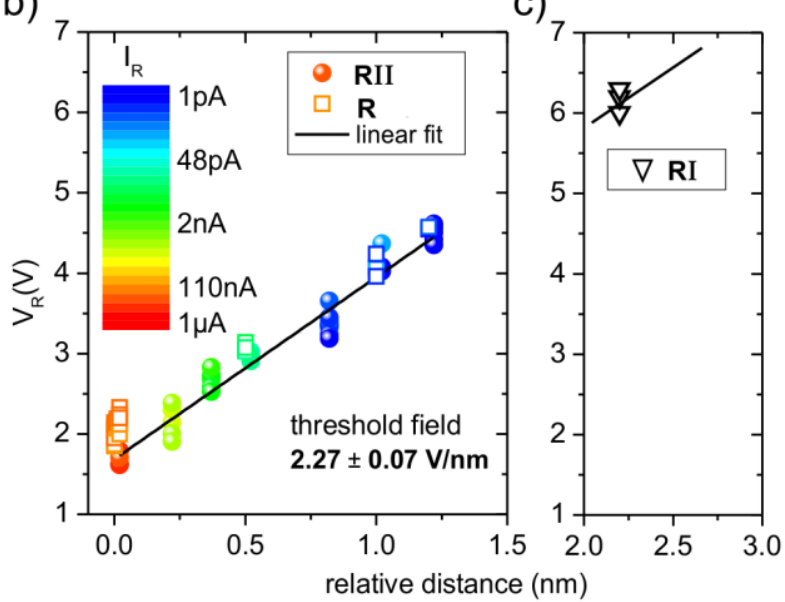

Figure 3. Experimental evidence for the electric-field-stimulated reaction. a) $I(V)$ curves recorded at relative distances $z$ from the height where the feedback loop was switched off and approached by $200 \mathrm{pm}$. The origin of the $z$ axis corresponds to a tunneling resistance of 16.5 $\pm 1.2 \mathrm{M} \Omega$. The sudden change in the current traces at which the reaction occurs is marked by $\left(I_{R}, V_{R}\right)$. b) The threshold voltage $V_{R}$ at which the reaction pathways $\mathbf{R}$ (1-2 transition) and RII (1i-2 transition) are activated, respectively, follow a linear dependence with $z$, a behavior which is characteristic of an electric-field-driven reaction. The threshold current, $I_{R}$, is depicted in the color map. c) At larger tip-sample distances, with the tip withdrawn by more than $2 \mathrm{~nm}$, the electric field stimulates the pathway RI (1-1i transition) only. The current at these large tip-sample distances is of the order of the noise level of $0.1 \mathrm{pA}$. 
The adsorption of thiophene, or other molecules containing the thiophene moiety, on different metallic surfaces is known to cause a reduction of the activation barrier for the desulfurization reaction, which has been shown to be thermally stimulated at room temperature and above. ${ }^{35-37}$ In our case, the adsorption of tetracenothiophene on $\mathrm{Cu}(111)$ also lowers this activation barrier. Here, we have demonstrated the use of an electric field as stimulus at cryogenic temperatures to overcome the remaining barrier for the direct desulfurization reaction.

The first step RI is triggered by the electric field oriented from the sample towards the tip, practically in the absence of tunneling electrons. The induced electron density of the adsorbed molecule in the initial state 1 (Figure 4a) shows an electron transfer from the surface to the entire molecule that corresponds approximately to one additional electron populating the LUMO of the adsorbed molecule, i.e., essentially a TCT anion. This surface-modifiedLUMO, shown in Figure 4b, does not show typical bonding character with respect to the $\mathrm{C}-\mathrm{S}-\mathrm{C}$ bonds, as it is reflected in the absence of electronic charge at the position of the $\mathrm{S}$ atom. The interaction with the applied electric field disturbs this arrangement in the vicinity of the tip position and may lower the energy barrier of the reaction. In addition, it acts on the $\mathrm{S}-\mathrm{C}$ dipole of the TCT anion while leading to a weakening of the sulfur-carbon bond. According to a Bader charge analysis ${ }^{38-40}$ the positive charge on the $\mathrm{S}$ atom is +0.2 e and the negative charge on the two neighboring $\mathrm{C}$ atoms are -0.29 e and -0.16 e. The positively charged $\mathrm{S}$ atom is attracted towards the tip, while the two negatively charged neighboring $\mathrm{C}$ atoms are repelled under the applied electric field, leading to an elongation of the $\mathrm{C}-\mathrm{S}$ bonds and, eventually, to the breakdown of one of them. The exact process might be similar to the breaking of a S-C bond in a non-radiative decay of excited thiophenes. ${ }^{41}$ 


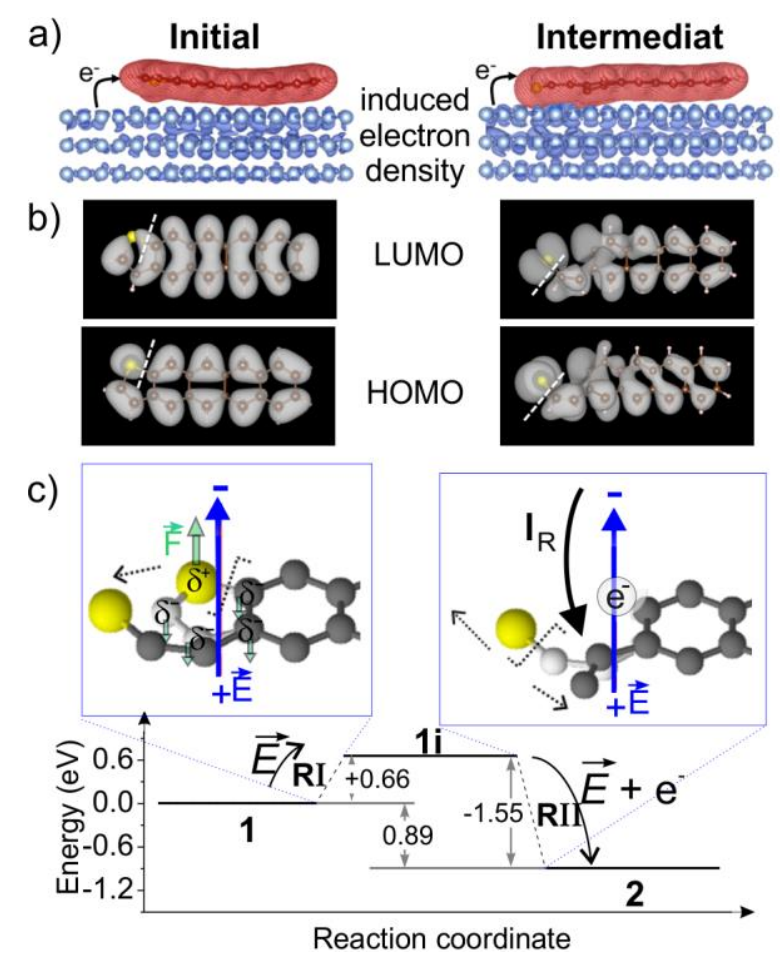

Figure 4. Effect of the electric field on the reaction mechanism. a) Induced electron density,

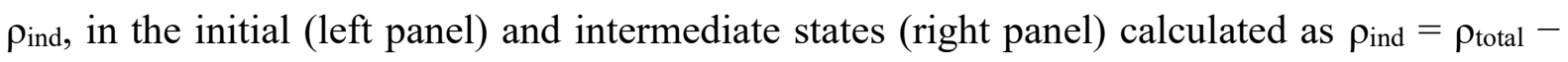
$\rho_{\text {mol }}-\rho_{\text {surf }}$ with $\rho_{\text {total }}$ the charge density of the entire system, $\rho_{\text {mol }}$ the charge density of the molecule and $\rho_{\text {surf }}$ the charge density of the clean surface slab. Isovalues are $2 \times 10^{-2} \mathrm{e} / \mathrm{bohr}^{3}$ and $-2 \times 10^{-4} \mathrm{e} / \mathrm{bohr}^{3}$, respectively, displayed in red and blue. b) Surface-modified LUMO and HOMO of the initial (left panels) and intermediate states (right panels). c) Energetic pathway of the desulfurization reaction considering the total energy of each molecular conformation with respect to the initial state. In the final state, the expelled $\mathrm{S}$ atom is considered adsorbed in its equilibrium site on $\mathrm{Cu}(111)$ without interaction with the TCT molecular part. Insets: Ball-and stick representation superposing the molecular structures at the thiophene side before (light-gray) and after (dark-gray) the reaction RI - left panel and RII - right panel, respectively. The bond cleavage and the modifications of the position are drawn with dotted lines. The electric field and the tunneling current are schematized as a colored arrow (blue) 
and solid-black arrow, respectively. The charge distribution of the initial state and the forces acting on the system due to the presence of the electric field are indicated by green arrows.

The corresponding energies of the initial, intermediate and final state are shown in Figure 4c. An energy barrier for $\mathbf{R I}$ of about $0.9 \mathrm{eV}$ has been estimated using an improved nudged elastic band minimization (NEB) method called the climbing image method. ${ }^{42,43}$ Indeed, this finding is consistent with DFT calculations reporting modifications of $\mathrm{C}-\mathrm{S}$ bonds under electric fields ${ }^{44}$ and experimental evidence of processes induced by an electric field. ${ }^{16-19,25-27}$

The second reaction step RII is also driven with the same bias polarity. However, as already mentioned, it occurs only if both the electric field and the injection of electrons are present. Thus, the reaction is triggered by a combination of electronic excitations and electric field effects. The electric field with the correct polarity induces changes of the local charge distribution and modifies the energy barrier of the reaction, as in reaction RI. Additionally, the injection of electrons increases the population of the molecular orbital visualized at the Fermi level of the metal (Figure 2b). This orbital has clear antibonding character (Figure $4 a, b)$ and, therefore, increases the reaction rate for the scission of the remaining $\mathrm{C}-\mathrm{S}$ bond and the removal of the $\mathrm{S}$ atom. Alternatively, the injected electrons might couple to vibronic states of the molecule which consequently could cleave the remaining $\mathrm{C}-\mathrm{S}$ bond. This reaction is exothermic by about $0.89 \mathrm{eV}$ (Figure 4c), which means that the sulfur atom has sufficient energy, compared to diffusion barriers of about $0.25 \mathrm{eV}$ for $\mathrm{S} / \mathrm{Cu}(111),{ }^{45}$ to diffuse on the surface before reaching its equilibrium adsorption state on the $\mathrm{Cu}$ surface. 


\section{CONCLUSION}

Our investigations reveal the two-step chemical pathway of the direct desulfurization of tetracenothiophene molecules adsorbed on a $\mathrm{Cu}(111)$ surface. The dissociation reaction is triggered locally by an electric field applied to the confined tunnel junction between the STM tip and the thiophene unit. Experimental control at the atomic level and comparison with theoretical calculations resolve the two elementary steps of this direct desulfurization reaction. The detailed experimental analysis of the stimuli involved to initiate the chemical reaction suggest that the first step, dissociation of one of the two $\mathrm{C}-\mathrm{S}$ bonds, is activated solely by the electric field, whereas the second step, the breaking of the remaining $\mathrm{C}-\mathrm{S}$ bond, is a consequence of the two driving forces electric field and injection of electrons. Triggering chemical processes by applying an electric field to a specific molecular moiety highlights the high potential of oriented external electric fields for single-molecule chemistry. ${ }^{30}$

\section{METHODS}

\section{Synthesis}

Tetraceno[2,3-b]thiophene (TCT) was synthesized from tetraceno[2,3-b]thiophene-5,12dione. ${ }^{46}$ First, thiophene-2,3-dicarbaldehyde was obtained from thiophene-3-carbaldehyde (0.98 $\mathrm{g}, 7 \mathrm{mmol})$ and then condensed with 1,4-dihydroxyanthracene (1.46 $\mathrm{g}, 7 \mathrm{mmol})$, obtained from 1,4-anthracenedione, to yield the corresponding tetracenothiophene quinone. The aldol condensation reaction was conducted in a mixture of ethanol and tetrahydrofuran using $15 \%$ aqueous solution of sodium hydroxide as a base. The quinone (1.0 g, $3.18 \mathrm{mmol})$ was then reduced to tetraceno[2,3-b]thiophene using sodium borohydride $\left(\mathrm{NaBH}_{4}, 1.2 \mathrm{~g}\right.$, $31.85 \mathrm{mmol}, 10 \mathrm{eq})$ in anhydrous tetrahydrofuran $(60 \mathrm{ml})$ for the reduction to the diol, 
followed by deoxygenation with tin(II)chloride $\left(\mathrm{SnCl}_{2}, 3.59 \mathrm{~g}, 15.9 \mathrm{mmol}, 5 \mathrm{eq}\right) / 10 \%$ aqueous solution of $\mathrm{HCl}$. Finally, tetraceno[2,3-b]thiophene was purified by sublimation.

\section{Experimental details}

The experiments were performed under ultrahigh-vacuum (UHV) conditions. The $\mathrm{Cu}(111)$ substrate was cleaned by standard procedures of Ar+ ion bombardment and subsequent annealing at $800 \mathrm{~K}$. The TCT molecules were thermally sublimated from a ceramic crucible at $485 \mathrm{~K}$ onto the $\mathrm{Cu}(111)$ surface held at 200 - $250 \mathrm{~K}$ during the deposition. Subsequently, the sample was transferred in situ into the STM operating at low temperatures $(6 \mathrm{~K})$. The STM measurements were carried out by applying the bias voltages to the sample in both constant-current and constant-height modes. Submolecular resolution imaging was achieved by placing a TCT molecule at the apex of the tip in a vertical manipulation procedure. The relative tip-sample distance is referred to the following set point. The STM tip was stabilized at $100 \mathrm{pA}, 100 \mathrm{mV}$ above the thiophene ring of the molecules in the initial state $\mathbf{1}$. Then, the feedback loop was switched off and the tip was approached towards the surface by $200 \mathrm{pm}$. The tunneling resistance at this position is $16.5 \pm 1.2 \mathrm{M} \Omega$. This new tip-sample distance we defined as the origin of our z-axis.

\section{Computational details}

DFT-vdW calculations were performed using the VASP code. ${ }^{47,48}$ To model the $\mathrm{TCT} / \mathrm{Cu}(111)$ system, a 4 layer slab $8 \times 6$ periodic supercell was employed. Ion-electron interaction was described with the projector augmented-wave (PAW) method ${ }^{49}$ and exchange correlation was modeled within the generalized gradient approximation (GGA). ${ }^{50}$ Van-derWaals dispersion forces were included using the vdW-DF-cx method. ${ }^{31,32}$ We considered a $500 \mathrm{eV}$ energy cutoff in the plane wave expansion, with a $2 \times 3 \mathrm{k}$-point mesh in the $1 \times 1$ unit cell as sampling of the Brillouin zone reciprocal space. Electronic convergence criterion was 
$1 \times 10^{-4}$ for all static calculations and convergence on forces in the relaxations was 0.05 eV/Å. STM appearance simulations were computed in the Tersoff-Hamann approximation. ${ }^{33}$

\section{ASSOCIATED CONTENT}

Supporting Information. A detailed description of the adsorption conformation of TCT molecules on $\mathrm{Cu}(111)$ is included in the supporting file. This material is available free of charge via the Internet at http://pubs.acs.org.

\section{AUTHOR INFORMATION}

\section{Corresponding Author}

*Bogdana Borca: bogdana.borca@infim.ro; Uta Schlickum: u.schlickum@fkf.mpg.de

\section{Present Addresses}

\# Institut des Sciences Analytiques et de Physico-chimie pour 1'Environnement et les Matériaux (IPREM) UMR 5254, 64053 Pau, France

$\dagger$ Karlsruher Institut für Technologie - KIT, Engesserstrasse 13 , 76131 Karlsruhe, Germany

$\perp$ Stanford University, Stanford, California 94305, USA

\section{Author Contributions}

$\$$ Bogdana Borca, Tomasz Michnowicz, and Rémi Pétuya contributed equally.

\section{Funding Sources}

Emmy-Noether-Program of the Deutsche Forschungsgemeinschaft, the SFB 767, Basque Departamento de Universidades e Investigación (grant no. IT-756-13) and the Spanish Ministerio de Economía y Competitividad (grant no. FIS2013-48286-C2-8752-P and FIS2016-75862-P). 


\section{ACKNOWLEDGMENT}

We acknowledge funding by the Emmy-Noether-Program of the Deutsche Forschungsgemeinschaft and the SFB 767. R.P. and A.A. thank the Basque Departamento de Universidades e Investigación (grant no. IT-756-13) and the Spanish Ministerio de Economía y Competitividad (grant no. FIS2013-48286-C2-8752-P and FIS2016-75862-P) for financial support. The authors thank M. Ternes for fruitful discussions.

\section{REFERENCES}

1. Falkowski, P. G.; Fenchel, T.; Delong, E. F. The Microbial Engines That Drive Earth's Biogeochemical Cycles. Science 2008, 320, 1034-1038.

2. Wortmann, U. G.; Paytan, A. Rapid Variability of Seawater Chemistry. Science 2012, 337, 334-336.

3. Zhelezinskaia, I.; Kaufman, A. J.; Farquhar, J.; Cliff, J. Large Sulfur Isotope Fractionations Associated with Neoarchean Microbial Sulfate Reduction. Science 2014, 346, 742-744.

4. Babich, I. V.; Moulijn J. A. Science and Technology of Novel Processes for Deep Desulfurization of Oil Refinery Streams: a Review. Fuel 2003, 82, 607-631.

5. H. Topsøe, B. S.; Clausen, F.E.; Massoth, Catalysis Science and Technology vol. 11, Anderson, J. R.; Boudart, M., Eds.; Springer-Verlag, New York, 1996, 1-269.

6. Gary, J. H.; Handwerk, G. E.; Kaiser, M. J. Petroleum Refining: Technology and Economics, Fifth Edition; CRC press, 2007. 
7. Lee, H. J.; Ho, W. Single-Bond Formation and Characterization with a Scanning Tunneling Microscope. Science 1999, 286, 1719-1722.

8. Hla, S.-W.; Bartels, L.; Meyer, G.; Rieder, K.-H. Inducing All Steps of a Chemical Reaction with the Scanning Tunneling Microscope Tip: Towards Single Molecule Engineering. Phys. Rev. Lett. 2000, 85, 2777-2780.

9. Ohmann, R.; Vitali, L.; Kern, K. Actuated Transitory Metal - Ligand Bond as Tunable Electromechanical Switch. Nano Lett. 2010, 10, 2995-3000.

10. Mohn, F.; Repp, J.; Gross, L.; Meyer, G.; Dyer, M. S.; Persson, M. Reversible Bond Formation in a Gold-Atom-Organic-Molecule Complex as a Molecular Switch. Phys. Rev. Lett. 2010, 105, 266102-266105.

11. Liljeroth, P.; Repp, J.; Meyer, G. Current-Induced Hydrogen Tautomerization and Conductance Switching of Naphthalocyanine Molecules. Science 2007, 317, 1203-1206.

12. Simic-Milosevic, V.; Mehlhorn, M.; Rieder, K.-H.; Meyer, J.; Morgenstern, K. Electron Induced Ortho-Meta Isomerization of Single Molecules. Phys. Rev. Lett. 2007, 98, 116102-14.

13. Maksymovych, P.; Sorescu, D. C.; Jorda, K. D.; Yates, J. T. Jr.; Collective Reactivity of Molecular Chains Self-Assembled on a Surface. Science 2008, 322, 1664-1667.

14. Néel, N. ; Lattelais, M. ; Bocquet, M.-L.; Kröger, J. Depopulation of SinglePhthalocyanine Molecular Orbitals upon Pyrrolic-Hydrogen Abstraction on Graphene, ACS Nano 2016, 10, 2010-2016.

15. Shen, T. C.; Wang, C.; Abeln, G. C.; Tucker, J. R. Atomic-Scale Desorption Through Electronic and Vibrational Excitation Mechanisms. Science 1995, 268, 1590-1592. 
16. Serrate, D.; Moro-Lagares, M.; Piantek, M.; Pascual, J. I.; Ibarra, M. R. Enhanced Hydrogen Dissociation by Individual Co Atoms Supported on $\operatorname{Ag}(111)$. J. Phys. Chem. C 2014, 118, 5827-5832.

17. Minato, T.; Kajita, S.; Pang, C. L.; Asao, N.; Yamamoto, Y.; Nakayama, T.; Kawai, M.; Kim, Y. Tunneling Desorption of Single Hydrogen on the Surface of Titanium Dioxide. ACS Nano 2015, 9, 6837-6842.

18. Qiu, X. H.; Nazin, G. V.; Ho, W. Mechanisms of Reversible Conformational Transitions in a Single Molecule. Phys. Rev. Lett. 2004, 93, 196806-1-4.

19. Alemani, M.; Peters, M. V.; Hecht, S.; Rieder, K.-H.; Moresco, F.; Grill, L. Electric Field Induced Isomerization of Azobenzene by STM. J. Am. Chem. Soc. 2006, 128, 14446-14447.

20. Lastapis, M.; Martin, M.; Riedel, D.; Hellner, L.; Comtet, G.; Dujardin, G. PicometerScale Electronic Control of Molecular Dynamics Inside a Single Molecule. Science 2005, $308,1000-1003$.

21. Iancu, V.; Deshpande, A.; Hla, S.-W. Manipulating Kondo Temperature via Single Molecule Switching. Nano Lett. 2006, 6, 820-823.

22. Henningsen, N.; Franke, K. J.; Torrente, I. F.; Schulze, G.; Priewisch, B.; Ru1ck-Braun, K.; Dokic, J.; Klamroth, T.; Saalfrank, P.; Pascual, J.I. Inducing the Rotation of a Single Phenyl Ring with Tunneling Electrons. J. Phys. Chem. C 2007, 111, 14843-14848.

23. Simic-Milosevic, V.; Morgenstern, K. Bending a Bond within an Individual Adsorbed Molecule. J. Am. Chem. Soc. 2009, 131, 416-417.

24. Wang, Y.; Kröger, J.; Berndt, R.; Hofer, W. A. Pushing and Pulling a Sn Ion through an Adsorbed Phthalocyanine Molecule. J. Am. Chem. Soc. 2009, 131, 3639-3643. 
25. Grill, L.; Rieder, K.-H.; Moresco, F. Exploring the Interatomic Forces between Tip and Single Molecules during STM Manipulation. Nano Lett. 2006, 6, 2685-2689.

26. Grill, L.; Rieder, K.-H.; Moresco, F.; Rapenne, G.; Stojkovic, S.; Bouju, X.; Joachim, C. Rolling a Single Molecular Wheel at the Atomic Scale. Nat. Nanotechnol. 2007, 2, 95-98.

27. Kudernac, T.; Ruangsupapichat, N.; Parschau, M.; Maciá, B.; Katsonis, N.; Harutyunyan, S. R.; Ernst, K.-H.; Feringa, B. L. Electrically Driven Directional Motion of a Four-Wheeled Molecule on a Metal Surface. Nature, 2011, 479, 208-211.

28. Pavliček, N.; Schuler, B.; Collazos, S.; Moll, N.; Pérez, D.; Guitián, E.; Meyer, G.; Peña, D.; Gross, L. On-Surface Generation and Imaging of Arynes by Atomic Force Microscopy. Nat. Chem. 2015, 7, 623-628.

29. Aragonès, A. C.; Haworth, N. L.; Darwish, N.; Ciampi, S.; Bloomfield, N. J.; Wallace, G. G.; Diez-Perez, I. ; Coote, M. L. Electrostatic Catalysis of a Diels-Alder Reaction. Nature 2016, 531, 88-91.

30. Shaik, S.; Mandal. D.; Ramanan, R. Oriented Electric Fields as Future Smart Reagents in Chemistry. Nat. Chem. 2016, 8, 1091-1098.

31. Berland, K; Hylgaard, P. Exchange Functional that Tests the Robustness of the Plasmon Description of the van der Waals Density Functionals. Phys. Rev. B. 2014, 89, 035412-1-8.

32. Björkman, T. Testing Several Recent van der Waals Density Functionals for Layered Structures. J. Chem. Phys. 2014, 141, 074708-1-6.

33. Tersoff, J.; Hamann, D.R. Theory and Application for the Scanning Tunneling Microscope. Phys. Rev. Lett. 1996, 77, 3865-3868. 
34. Borca, B.; Schendel, V.; Pétuya, R.; Pentegov, I.; Michnowicz, T.; Kraft, U.; Klauk, H.; Arnau, A.; Wahl, P.; Schlickum, U.; Kern, K. Bipolar Conductance Switching of Single Anthradithiophene Molecules. ACS Nano 2015, 9, 12506-12512.

35. Stöhr, J.; Gland, J. L.; Kollin, E. B.; Koestner, R. J.; Johnson, A. L.; Muetterties, E. L.; Sette, F. Desulfurization and Structural Transformation of Thiophene on the Pt (111) Surface. Phys. Rev. Lett. 1984, 53, 2161-2164.

36. Dinca, L. E.; MacLeod, J. M.; Lipton-Duffin, J.; Fu, C.; Ma, D.; Perepichka, D. F.; Rosei, F. Tailoring the Reaction Path in the On-Surface Chemistry of Thienoacenes. J. Phys. Chem. C 2015, 119, 22432-22438.

37. Dinca, L.E.; Fu, C.; MacLeod, J.M.; Lipton-Duffin, J.; Brusso, J.L.; Szakacs, C.E.; Ma, D.; Perepichka, D.F.; Rosei, F. Unprecedented Transformation of Tetrathienoanthracene into Pentacene on Ni(111). ACS Nano 2013, 7, 1652-1657.

38. Henkelman, G.; Arnaldsson, A.; Jónsson, H. A., Fast and Robust Algorithm for Bader Decomposition of Charge Density, Comput. Mater. Sci. 2006, 36, 254-360.

39. Sanville, E.; Kenny, S. D.; Smith, R.; Henkelman, G., An Improved Grid-Based Algorithm for Bader Charge Allocation, J. Comp. Chem. 2007, 28, 899-908.

40. Tang, W.; Sanville, E.; Henkelman, G. A., Grid-Based Bader Analysis Algorithm without Lattice Bias, J. Phys.: Condens. Matter 2009, 21, 084204-1-7.

41. Salzmann, S.; Kleinschmidt, M.; Tatchen, J.; Weinkauf, R.; Marian, C.M.. Excited States of Thiophene: Ring Opening as Deactivation Mechanism. Phys. Chem. Chem. Phys. 2008, 10, 380-392. 
42. Henkelman, G.; Jónsson, H. A., Climbing Image Nudged Elastic Band Method for Finding Saddle Points and Minimum Energy Paths, J. Chem. Phys. 2000, 113, 9901-9904

43. Henkelman, G.; Jónsson, H., Improved Tangent Estimate in the Nudged Elastic Band Method for Finding Minimum Energy Paths and Saddle Points. J. Chem. Phys. 2000, 113, 9978-9985.

44. Ye, Y.; Zhang, M.; Liu, H.; Liu, X.; Zhao, J. Theoretical Investigation on the Oligothienoacenes under the Influence of External Electric Field. J. Phys. Chem. Solids 2008, $69,2615-2621$.

45. Barth, J. V. Transport of Adsorbates at Metal Surfaces: from Thermal Migration to Hot Precursors. Surf. Sci. Rep. 2000, 40, 75-149.

46. Kraft, U.; Anthony, J. E.; Ripaud, E.; Loth, M. A.; Weber, E.; Klauk, H. Low-Voltage Organic Transistors Based on Tetraceno[2,3-b]thiophene: Contact Resistance and Air Stability. Chem. Mater. 2015, 27, 998-1004.

47. Kresse, G.; Hafner, J. Ab Initio Molecular-Dynamics Simulation of the Liquid-MetalAmorphous-Semiconductor Transition in Geranium. Phys. Rev. B 1994, 49, 14251-14269.

48. Kresse, G.; Furthmuller, J. Efficient Iterative Schemes for Ab Initio Total-Energy Calculations Using a Plane-Wave Basis Set. Phys. Rev. B 1996, 54, 11169-11186.

49. Blöchl, P. E. Projector Augmented-Wave Method. Phys. Rev. B 1994, 50, 17953-17979.

50. Perdew, J. P.;Burke, K.; Ernzerhof, M. Generalized Gradient Approximation Made Simple Phys. Rev. Lett. 1996, 77, 3865. Erratum. Phys. Rev. Lett. 1997, 78, 1396-1396. 
Table of Contents Graphic

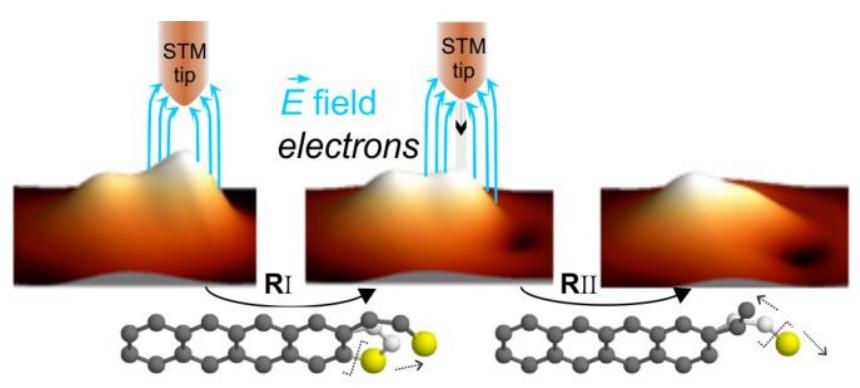

